\title{
Direct Numerical Simulation of Laminar Natural Convection in a Square Cavity at Different Inclination Angle
}

\author{
Mohammad Ilias Inam* \\ Department of Mechanical Engineering, Khulna University of Engineering \& Technology, Khulna-9203, BANGLADESH
}

Received: March 28, 2020, Revised: April 03, 2020, Accepted: April 03, 2020, Available Online: April 05, 2020

\begin{abstract}
The effect of angle of rotation on laminar natural convection inside the square cavity have been observed in this research. It was assumed that left and right walls heated isothermally, whereas other two walls act as adiabatic. This problem was solved by assuming 2-D and by Direct Numerical Simulation (DNS) method using ANSYS Fluent 16.0. A series of DNS simulation were carried out for different inclination angle $\left(\theta=0^{\circ} \sim 90^{\circ}\right)$ of the cavity at $R a=10^{3} \& 10^{4}$. It was observed that at average Nusselt number increase up to some value of angle of inclination after that it decrease though this variation is not significant.
\end{abstract}

Keywords: Natural Convection; Inclination Angle; Nusselt Number

This work is licensed under a Creative Commons Attribution-NonCommercial 4.0 International

\section{Introduction}

Study on natural convection heat transfer in square cavity have intense research interest due to its enormous engineering application like as building insulation, cooling devices for electronic instruments, nuclear reactor design and solar energy collectors etc. An extensive research have been conducted on this field by analytically, numerically and experimentally.

Natural heat transfer characteristics inside the enclosure is controlled by some parameter, like as shape of the enclosure, aspect ratio, Rayleigh number and angle of inclination etc. Extensive study have been conducted to understand the effect of shape, aspect ratio of enclosure. Numerical investigation of the heat transfer characteristics of two dimensional rectangular enclosure with isothermal boundary condition for different aspect ratio was studied by Churchill [1] and De Vahl Davis [2]. An extensive review of natural convection inside the enclosure (non-square) were carried out by Das et al. [3]. The fluid flow due to the induce buoyancy force becomes turbulent when Rayleigh number exceed by some critical values [4]-[6]. Effect of inclination of rectangular cavity on the natural convection was studied by Sharif and Liu [7] and Kuyper et al. [8]. They conducted their simulation at high Rayleigh number.

It is observed form the previous literature review that there is a lack of understanding about the effect of angle of inclination of square cavity at low Rayleigh number, which motivates for this research. For these reason, a series of two dimensional Direct Numerical Simulation (DNS) were carried for different angle of rotation $\left(\theta=0^{\circ} \sim 90^{\circ}\right)$ of square cavity at low Rayleigh number $\left(R a=10^{3} \& 10^{4}\right)$ using ANSYS Fluent 16.0.

\section{Methodology}

The physical system consider for this simulation is a two dimensional square enclosure with dimension $(100 \mathrm{~mm} \times$ $100 \mathrm{~mm}$ ), containing Newtonian fluid, sketched at Fig. 1. The left wall was consider as a rigid wall with constant temperature equal to $310 \mathrm{~K}$ and right wall was consider as rigid wall with constant temperature equal to $300 \mathrm{~K}$. Other two walls were assumed as adiabatic wall. To observe the effect of angle inclination on the natural convection in a rectangular square cavity, the gravitational direction was change during this simulation, instead of rotating the square cavity. For zero degree rotation $\left(\theta=0^{\circ}\right)$, gravity was considered acting along the negative $y$-axis. However, for any arbitrary rotation $\theta$, the component of gravitation forces were considered to be equal to $-g \sin \theta$ and $-g \cos \theta$ which is acting along $x$ - axis and $y$-axis, respectively. During this simulation it was assumed that flow was steady, incompressible and two dimensional. Also Boussinesq approximation was considered during these DNS simulation run.

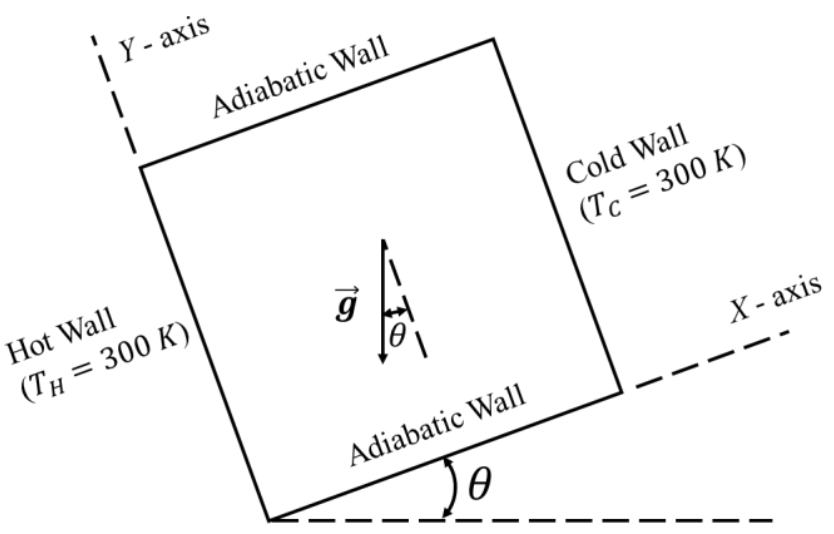

Fig. 1 Domain with boundary condition

The two dimensional governing equations (Continuity, Navier-Stokes equation and Energy equation) for these flow in Cartesian coordinates are written as follows:

$\frac{\partial u}{\partial x}+\frac{\partial v}{\partial y}=0$

$u \frac{\partial u}{\partial x}+v \frac{\partial u}{\partial y}=-\frac{1}{\rho_{\text {ref }}} \frac{\partial P}{\partial x}+\vartheta\left(\frac{\partial^{2} u}{\partial x^{2}}+\frac{\partial^{2} v}{\partial y^{2}}\right)-\frac{\rho}{\rho_{\text {ref }}} g \sin \theta$

$u \frac{\partial v}{\partial x}+v \frac{\partial v}{\partial y}=-\frac{1}{\rho_{\text {ref }}} \frac{\partial P}{\partial y}+\vartheta\left(\frac{\partial^{2} u}{\partial x^{2}}+\frac{\partial^{2} v}{\partial y^{2}}\right)-\frac{\rho}{\rho_{\text {ref }}} g \cos \theta$ 
$u \frac{\partial T}{\partial x}+v \frac{\partial T}{\partial y}=\alpha\left(\frac{\partial^{2} T}{\partial x^{2}}+\frac{\partial^{2} T}{\partial y^{2}}\right)$

where $\mathrm{u}, \mathrm{v}$ are the velocity component in the $\mathrm{x}$ and $\mathrm{y}$ direction, respectively. Also, kinematic viscosity and thermal diffusivity represent by $\vartheta$ and $\alpha$, respectively.

The appropriate boundary conditions are:

Left wall: $u=v=0 \& T=310 K$

Right wall: $u=v=0 \& T=300 \mathrm{~K}$

Lower and upper wall: $u=v=0 \& \frac{d T}{d y}=0$

For these DNS simulations ANSYS Fluent 16.0 were used. ANSYS Fluent use finite volume method to discretize the above governing equations. Pressure based solver was used for these DNS simulation run. Also, SIMPLE scheme for pressure and velocity coupling; Green-Gauss cell based for gradient, PRESTO for pressure gradient and QUICK scheme for both momentum and energy equations.

In this paper, simulation were carried out for two different Rayleigh number $\left(\operatorname{Ra}=10^{3} \& 10^{4}\right)$, whereas this Rayleigh number is defined by the following equation:

$R a=\frac{g \beta\left(T_{H}-T_{C}\right) L^{3}}{\vartheta \alpha}$

Where, $\beta$ is thermal expansion coefficient, $L$ distance among hot and cold wall. For these simulation, consider air was the working fluid. The different value of $R a$ for these DNS simulation run was achieved by changing the value of gravitational acceleration, $g$. Also, Local Nusselt number, $\mathrm{Nu}$, and average Nusselt number, $N u_{a v}$, were calculated with the following equations:

$N u=\frac{h L}{k}$

$N u_{a v}=\frac{1}{L} \int_{0}^{L} N u d y$

Where, $h$ is the convection heat transfer coefficient and $k$ is the thermal conductivity.

A non-uniform structural mesh was created with ANSYS mesh with higher resolution near to the wall, see Fig. 2. Specifically, for these mesh generation both horizontal and vertical wall were divided by 100 division with center biasing type with bias factor 10 to create fine mesh along the wall. This result was validated by comparing with previous date which is listed in

Table 1. Converging criteria was set for these simulation based on average Nusselt number $\left(N u_{a v}\right)$ and simulation were run until this value of $N u_{a v}$ to become a constant. Fig. 3 depicts variation of average Nusselt number $\left(N u_{a v}\right)$ respect to number of iteration for $R a=10^{3}$ for zero degree angle of rotation. It is observed from Figure that average Nusselt number $\left(N u_{a v}\right)$ is changing significantly up to around 1000 , after that it becomes almost stable with little variation and reach to a constant value which equal to 1.118 at around 1650 number iteration. Once the value of $N u_{a v}$ reach to the constant value, this simulation were run for more number of iteration to ensure the steady nature of the result. It was observed from simulation that value of $N u_{a v}$ reach to the constant value when the value of the residual of continuity equation, $x$-velocity, $y$-velocity and energy equation reach to around $10^{-8}, 10^{-8}, 10^{-8}, 10^{-13}$, respectively. This procedure was followed for all the DNS runs carried out for this paper.

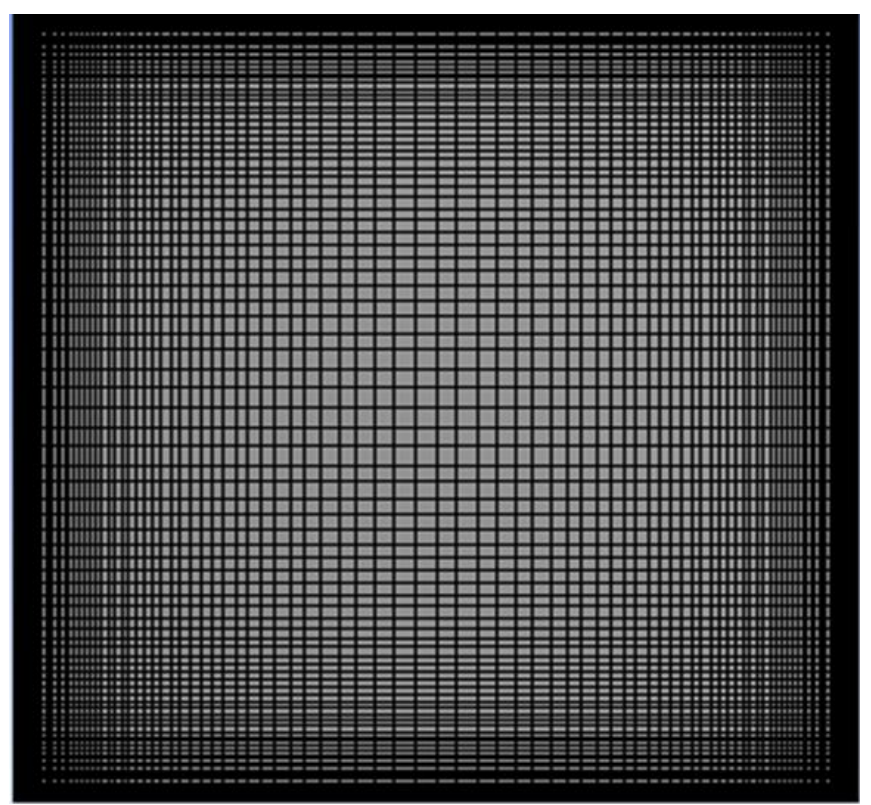

Fig. 2 Mesh used for these simulations

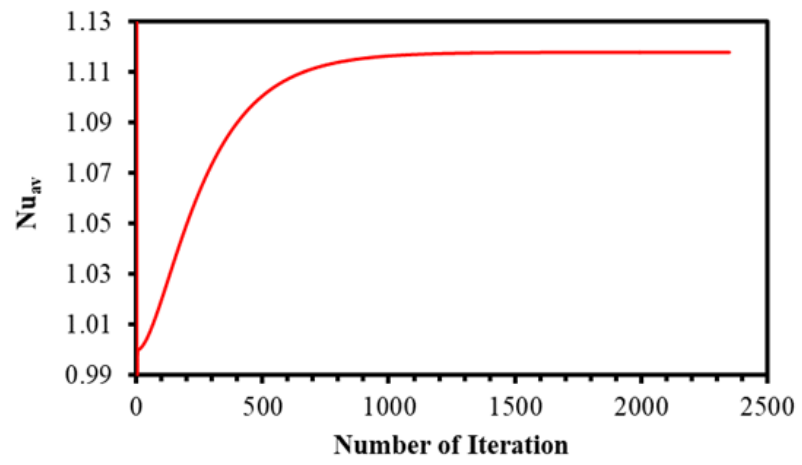

Fig. 3 Variation of average Nusselt number $\left(\mathrm{Nu}_{\mathrm{av}}\right)$ with number of iteration at $\mathrm{Ra}=10^{3} \& \theta=0$

Table 1 Comparison of present work with previous work for square cavity

\begin{tabular}{lcccc}
\hline & $R a=10^{3}$ & $R a=10^{4}$ & $R a=10^{5}$ & $R a=10^{6}$ \\
\hline Present study & 1.1179 & 2.2469 & 4.5347 & 8.8545 \\
De Vahl Davis and Jones (1983) & 1.118 & 2.243 & 4.519 & 8.799 \\
A. Rincón-Casado et. al. & 1.118 & 2.241 & 4.522 & 8.819
\end{tabular}




\section{Results and Discussion}

Fig. 4 depicts the temperature contour of the square cavity for different angle of rotation $(\theta)$ at $R a=10^{3}$. In this figure, temperature contour are shown without angle of inclination of the cavity. The exact position of the contour of the square cavity will be found by rotating anti-clock wise each contour shown in the figure with the value of $\theta$ is mention underneath of each figure. It is clearly observed form the figure that at $\theta=0^{\circ}$ at the top of the square cavity have higher temperature distribution for wider region compare to cooler region whereas in the bottom cooler region is wider than high temperature region. This result is expected due to the buoyancy effect. Fluid near to the left wall will rise due to the heating which reduce fluid density. Similarly, fluid density increase near to the right wall due to the cooling which produce a downward flow. Temperature distribution pattern are almost similar pattern for $\theta=0^{\circ} \sim 70^{\circ}$, whereas at $\theta=80^{\circ} \sim 90^{\circ}$ have different patter since gravitation force direction are almost perpendicular to the heating wall at $\theta=80^{\circ} \sim 90^{\circ}$, whereas it was parallel at $\theta=$ $0^{\circ}$.

Fig. 5 shows temperature contour of the square cavity for different angle of rotation $(\theta)$ at $R a=10^{4}$. In this figure, temperature contour are also shown without angle of inclination of the square cavity. The exact position of the contour of the square cavity will be found similarly by rotating each contour shown in the figure with the value of $\theta$ is mention.
It is clearly observed from figure that higher temperature zone is also wider in to top of square cavity and lower temperature zone is dominating at the bottom due to buoyancy effect which mention in the previous paragraph.

Fig. 6 depicts variation of local Nusselt number $(\mathrm{Nu})$ along the heated wall (left side wall) at different angle of inclination $(\theta)$ for $(a) R a=10^{3}$ and $(b) R a=10^{4}$. It is clearly seen from Fig. 6 ( $a$ ) for $R a=10^{3}$ at $\theta=0^{\circ}$ that $N u$ have higher value at the bottom of the heated wall which followed by slightly increase up to certain height and then starts to decrease and finally becomes almost constant near to the top of the wall. This similar behavior is also observed for other angle of inclination except $\theta=90^{\circ}$. The value of $N u$ is slightly higher at the lower side for $\theta=20^{\circ}$ which decrease at $\theta=50^{\circ}$ compare to $\theta=$ $0^{\circ}$, however no significant difference is observed at the upper sider of the heated wall. For $\theta=80^{\circ}, N u$ significantly decrease at the bottom of the wall and increase at the top of the wall. In case of $\theta=90^{\circ}, N u$ is equal to one along the whole heated wall since gravitation force acts to the perpendicular direction to the heated wall. Similar behavior is also observe for $R e=10^{4}$ for different angle of inclination except $\theta=90^{\circ}$, see Fig. $6(b)$. For $\theta=90^{\circ}$ at $\operatorname{Re}=10^{4}, N u$ have lower value at the bottom of the wall and higher at the top of the wall whereas as opposite behavior is observed for other angle of inclination. This is also happen since gravitation force acting to the parallel to the heated wall at $\theta=0^{\circ}$ which is changing respect to the angle of inclination and becomes perpendicular at $\theta=90^{\circ}$.

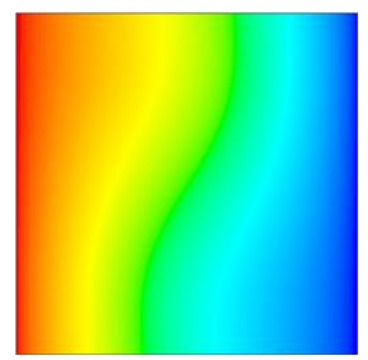

(a) $\theta=0^{\circ}$

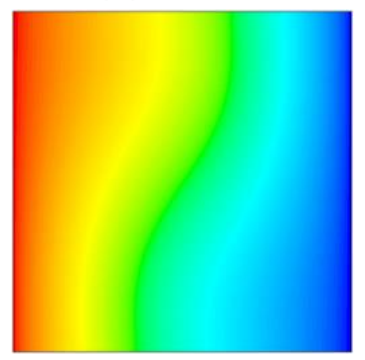

(e) $\theta=20^{\circ}$

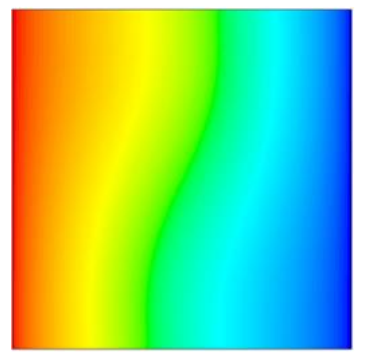

(i) $\theta=60^{\circ}$

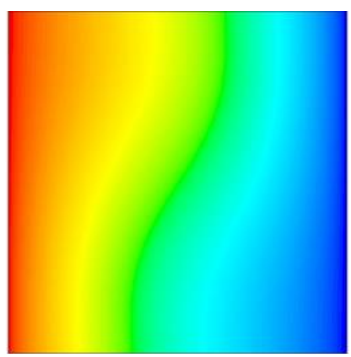

(b) $\theta=5^{\circ}$

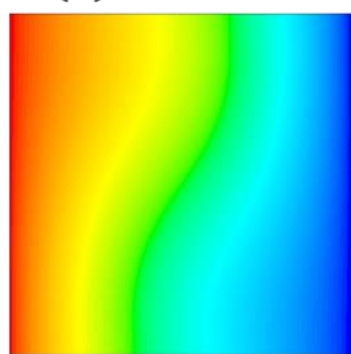

(f) $\theta=30^{\circ}$

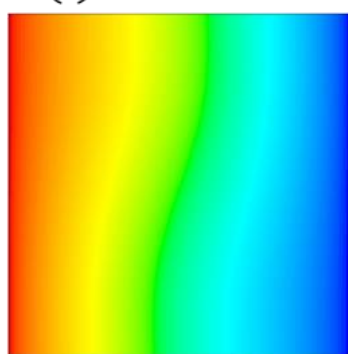

(j) $\theta=70^{\circ}$

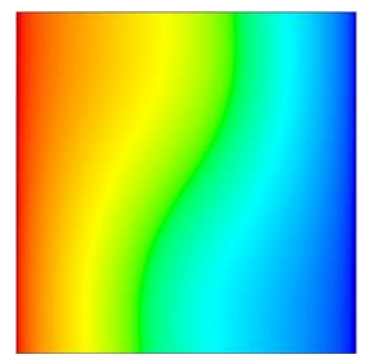

(c) $\theta=10^{\circ}$

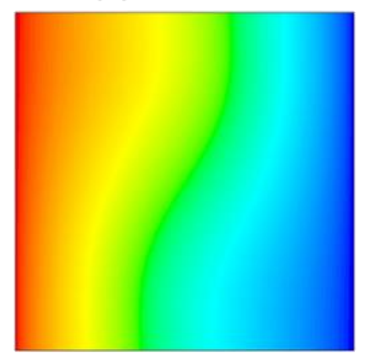

(g) $\theta=40^{\circ}$

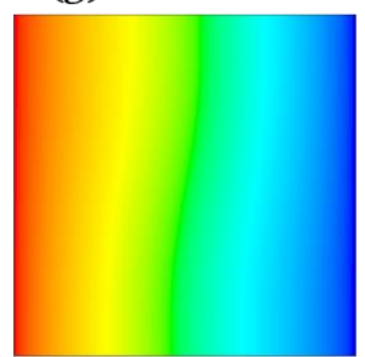

(k) $\theta=80^{\circ}$

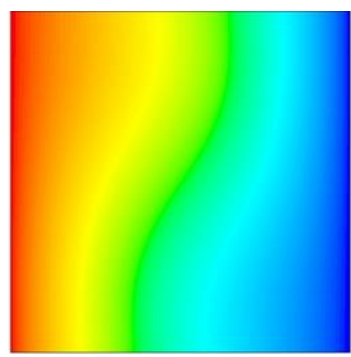

(d) $\theta=15^{\circ}$

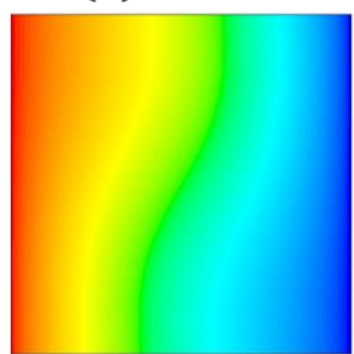

(h) $\theta=50^{\circ}$

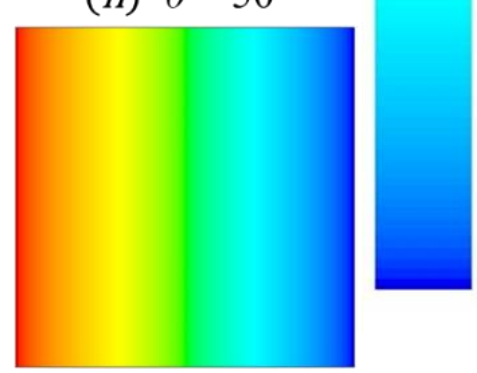

(k) $\theta=90^{\circ}$

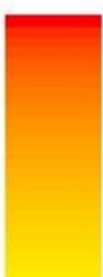

310

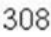

306

Fig. 4 Temperature contour of the square cavity for different angle of rotation $(\theta)$ at $\mathrm{Ra}=10^{3}$ 


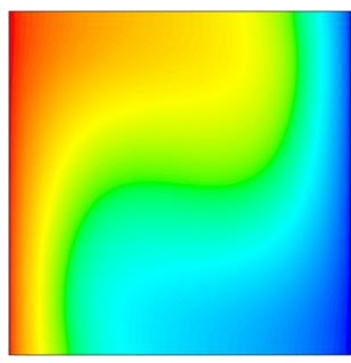

(a) $\theta=0^{\circ}$

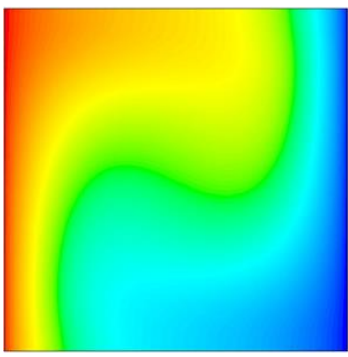

(e) $\theta=20^{\circ}$

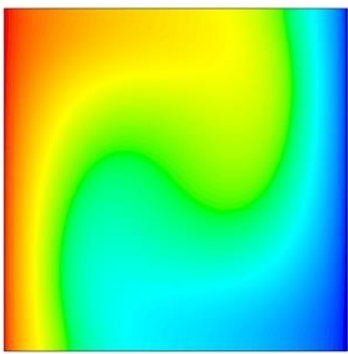

(i) $\theta=60^{\circ}$

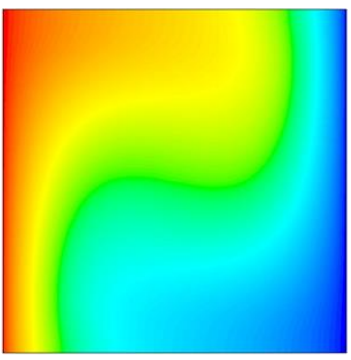

(b) $\theta=5^{\circ}$

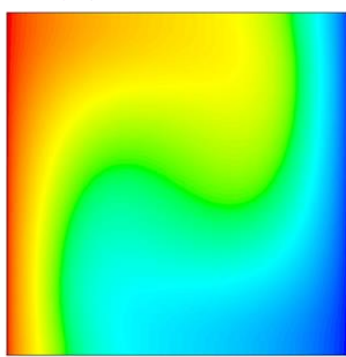

(f) $\theta=30^{\circ}$

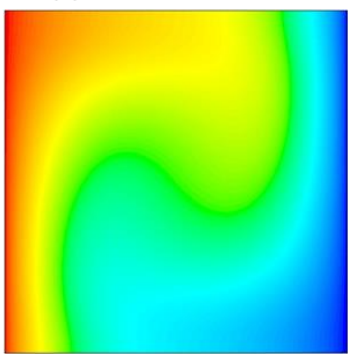

(j) $\theta=70^{\circ}$

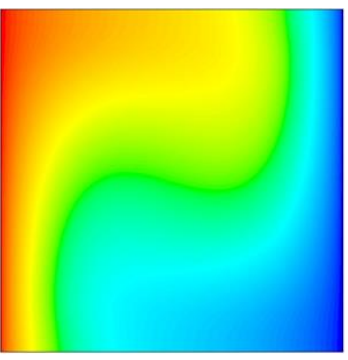

(c) $\theta=10^{\circ}$

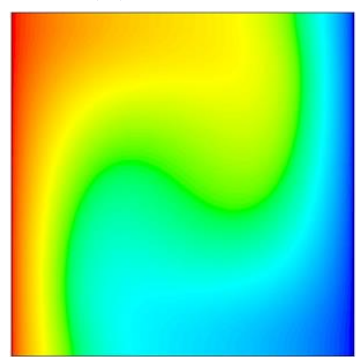

(g) $\theta=40^{\circ}$

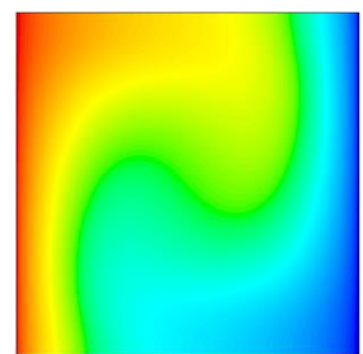

(k) $\theta=80^{\circ}$

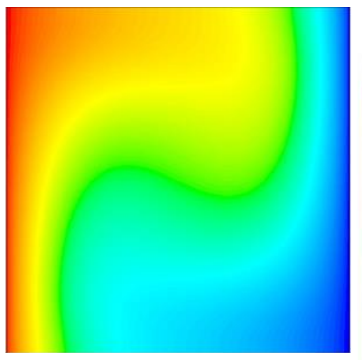

(d) $\theta=15^{\circ}$

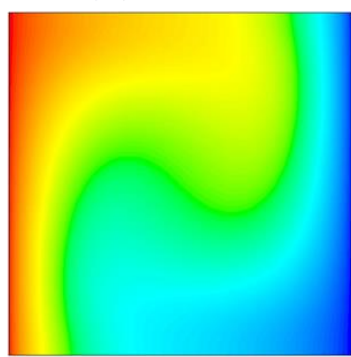

(h) $\theta=50^{\circ}$

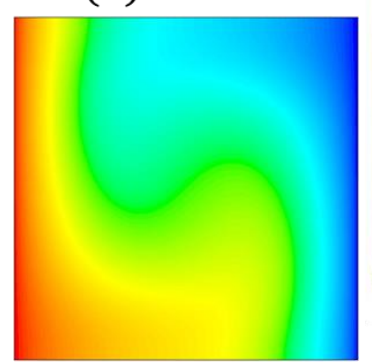

(k) $\theta=90^{\circ}$

Fig. 5 Temperature contour of inside the square cavity for different angle of rotation $(\theta)$ at $\mathrm{Ra}=10^{4}$

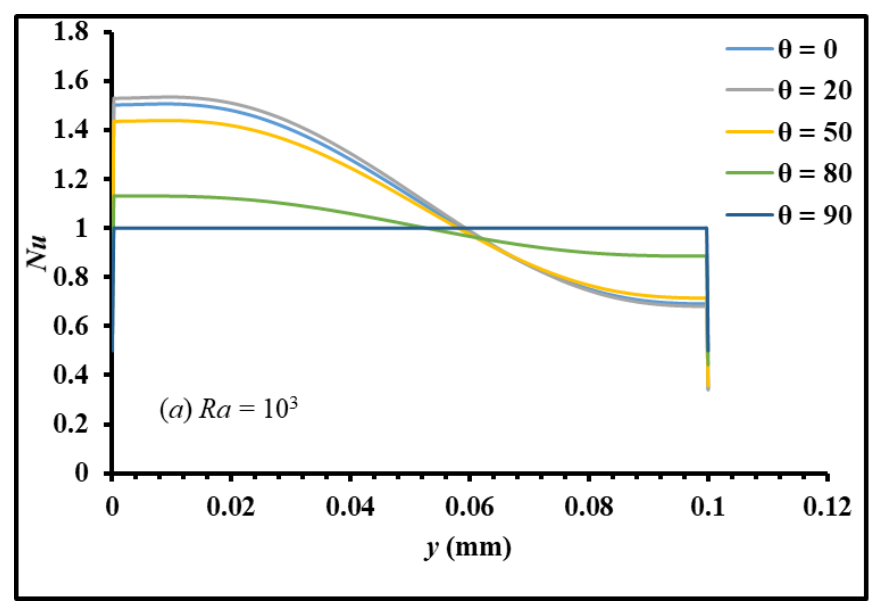

(a)

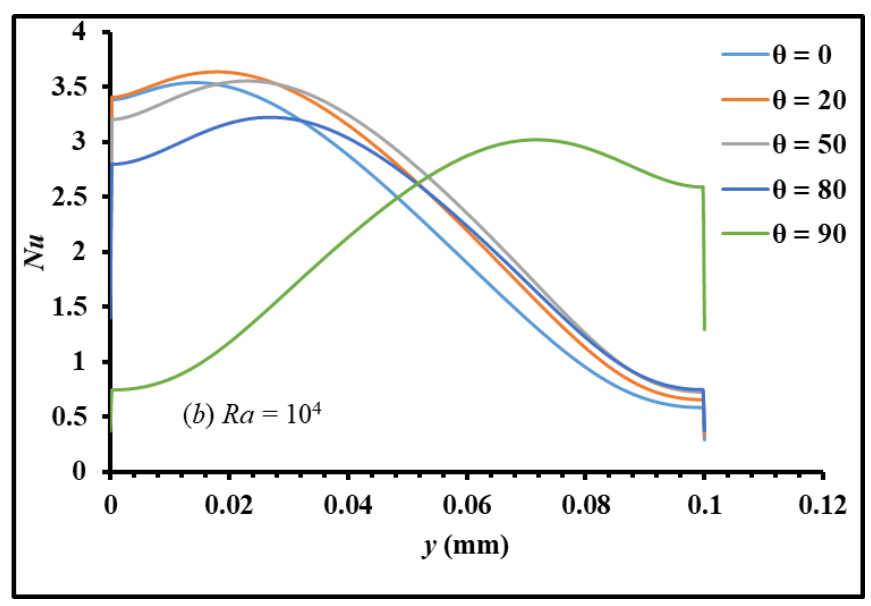

(b)

Fig. 6 Local Nusselt number $(\mathrm{Nu})$ variation along the left wall (heated wall) at different angle of inclination $(\theta)$ for $(\mathrm{a}) \mathrm{Re}=10^{3}$ and (b) $\operatorname{Re}=10^{4}$

Fig. 7 shows variation of average Nusselt number $\left(N u_{a v}\right)$ respect to angle of inclination $(\theta)$ for $R e=10^{3}$ and $R e=10^{4}$. It is clearly observed from figure that $N u_{a v}$ increase up to some angle of rotation than again decrease. For $\operatorname{Re}=10^{3}$, see Fig. 7 (a), $N u_{a v}$ increase up to $\theta=20^{\circ}$ by around $1.15 \%$ after that is decrease nonlinearly up to $\theta=90^{\circ}$ and reach to the unit value of average Nusselt number. When $R e=10^{4}$, see Fig. 7 (b), $N u_{a v}$ also increase up to $\theta=40^{\circ}$ by around $10.63 \%$ after that is decrease nonlinearly up to $\theta=90^{\circ}$. 


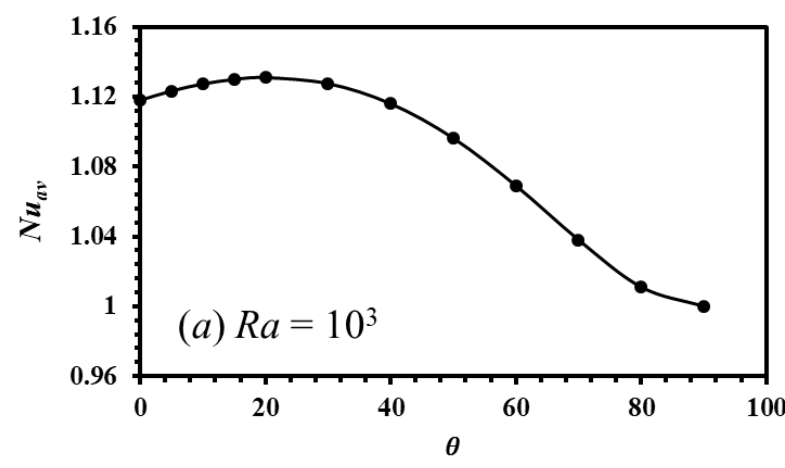

(a)

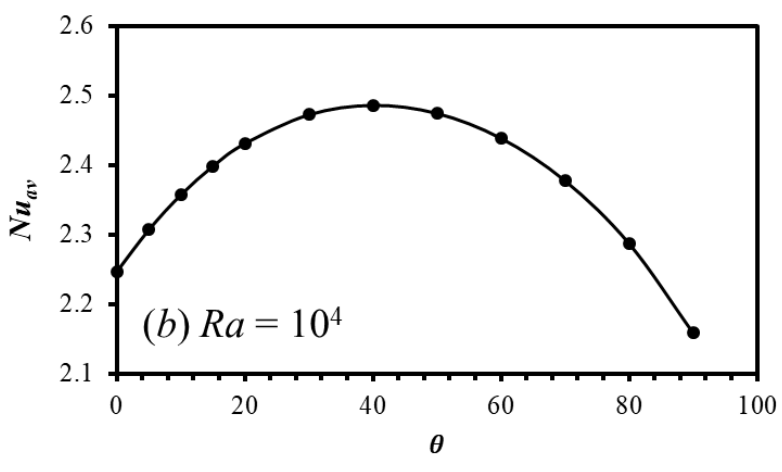

(b)

Fig. 7 Effect of angle of rotation $(\theta)$ on the average Nusselt number $\left(\mathrm{Nu}_{\mathrm{av}}\right)$ at (a) $\mathrm{Ra}=10^{3}$ and $(\mathrm{b}) \mathrm{Ra}=10^{4}$

\section{Conclusion}

This paper presents the effect of angle of orientation on the laminar natural convection inside a square cavity by DNS. It is observed that angle of orientation has positive effect on Average Nusselt number up to certain value, like as up to $\boldsymbol{\theta}=$ $\mathbf{2 0}^{\circ}$ for $\operatorname{Re}=10^{3}$ and $\boldsymbol{\theta}=\mathbf{4 0}^{\circ}$ for $R e=10^{4}$. Later on it decrease with increasing angle of orientation. However, the effect of angle of orientation of cavity has not much significant effect on Average Nusselt number.

\section{References}

[1] Wilkes JO, Churchill SW. The finite-difference computation of natural convection in a rectangular enclosure. AIChE Journal. 1966 Jan;12(1):161-6.

[2] de Vahl Davis G. Laminar natural convection in an enclosed rectangular cavity. International Journal of Heat and Mass Transfer. 1968 Nov 1;11(11):1675-93.
[3] Das D, Roy M, Basak T. Studies on natural convection within enclosures of various (non-square) shapes-A review. International Journal of Heat and Mass Transfer. 2017 Mar 1;106:356-406.

[4] Townsend AA. The structure of turbulent shear flow. Cambridge university press; 1980 Mar 6.

[5] Lesieur M. Turbulence in fluids: stochastic and numerical modelling. Boston, MA: Nijhoff; 1987 Jan 1.

[6] Foias C, Manley O, Rosa R, Temam R. Navier-Stokes equations and turbulence. Cambridge University Press; 2001 Aug 27.

[7] Foias C, Manley O, Rosa R, Temam R. Navier-Stokes equations and turbulence. Cambridge University Press; 2001 Aug 27.

[8] Sharif MA, Liu W. Numerical study of turbulent natural convection in a side-heated square cavityat various angles of inclination. Numerical Heat Transfer: Part A: Applications. 2003 May 1;43(7):693-716. 